\title{
A Cross-Cultural Values-Based Approach to the Diagnosis and Treatment of Dissociative (Conversion) Disorders
}

\author{
Anna Todeva-Radneva and Asen Beshkov
}

\subsection{Introduction}

Psychiatric diagnosis is in itself an art, incorporating the architectural accuracy of valid diagnostic criteria and the abstract poetry of patients' values and culture, which change the shapes and shades of every disorder. It is precisely these valueladen characteristics that many take to undermine the status of psychiatry as a science in the contemporary world of evidence-based medicine. However, in reality, they uncover the unrealized complexity of the nature of psychiatric conditions.

Dissociative (Conversion) disorders are some of the disorders presenting with the most polymorphic symptomatology. The difficulties of diagnosis arise not only in the necessity for numerous tests to exclude somatic (bodily) conditions (in the case of conversion predominantly neurological because of the frequent pseudoneurological presentation) but also in the fact that both the patient's (very often culturally rooted) personal understanding of and beliefs about the concept of "disease" can significantly alter the clinical presentation. This in turn may result in misinterpretation, misdiagnosis, and incorrect treatment. This is why it is of major significance for a doctor to take the time to understand the social and cultural background, as well as the contemporary social context and cultural milieu, of the patient and to use this understanding in the diagnostic process and in decisions about treatment.

As the following story of 'A.A' illustrates, when it comes to curing the person, bearing in mind the values and culture of the person in question, and not just the disease, means that medical treatments such as pharmacological therapies are usually not enough.

\footnotetext{
A. Todeva-Radneva $(\bowtie) \cdot$ A. Beshkov

Department of Psychiatry and Medical Psychology, Medical University of Plovdiv, Plovdiv, Bulgaria

e-mail: anna.todeva@mu-plovdiv.bg; asen.beshkov@mu-plovdiv.bg 


\subsection{A.A.: A Woman Trapped Not Only by Her Own Mind but also by the Invisible Boundaries Within Contemporary Medical Practice}

A.A. (not her real initials) is a 34-year-old Bulgarian woman of Romani descent, suffering from "Mixed dissociative (conversion) disorder". She has only elementary education and is unemployed. She lives with her husband, who she reports to be an abusive alcohol drinker with a tendency to aggressive behaviour when in an intoxicated state, and their two children.

A.A. has been admitted several times to the same psychiatric ward over the years with relapses of her condition. The main reason for the frequent relapses is lack of compliance with the prescribed therapy. Her medical and family histories were unremarkable before the onset of her illness and there is no evidence of physical or psychological trauma in her childhood and adolescence.

The first symptoms occurred after she lost a significant amount of weight in 2011 (35 kg over 9 months). She had no appetite and her mind was preoccupied with persistent thoughts of having stomach cancer. She felt on edge, anxious, futureless and tearful, and even admitted having thoughts of committing suicide.

In 2014 A.A. made a suicide attempt by cutting the veins in her wrists. Following this she was admitted to a hospital where she presented with anxiety, fear of various diseases and death, palpitations, shortness of breath, dizziness and faintness. The latter symptoms intensified even more her anxiety and the level of her fixation upon the significance of her symptoms. Whenever a "crisis" with autonomic nervous system symptoms occurred she would call an emergency unit.

The most recent hospitalization was in December 2018 when her condition worsened again. She presented with anxiety, fear of "going mad", tearfulness and numerous bodily symptoms, some of which she tried to fake. On admission her appearance was neglected, she kept underwear tied in a knot underneath her blouse, which according to her was charmed and protected her against black magic. She was also agitated, tense, spoke in a loud voice and could not stand still for a moment. Excoriation marks from attempts to hurt herself were visible. Her thinking process was accelerated but without delusions, and depersonalization phenomena were also noted. There was no insight on her part.

After all the tests necessary to exclude a number of possible bodily conditions, treatment was initiated with antidepressants (trazodone and escitalopram) and benzodiazepines (diazepam) but there was no improvement in her symptoms. On the tenth day, indeed, after starting treatment, her condition actually deteriorated-she expressed fears of dying, and had numbness and shortness of breath. After a thorough examination she admitted that she had spat out the medication given to her over the last 2 or 3 days. This led to the decision of disciplinary discharge from the hospital due to lack of compliance.

However, even though A.A's relatives were informed, they did not come to the hospital to take responsibility for her and her stay was in consequence prolonged. At that time she said that if she had been discharged she would have come back. During her extended stay another attempt to establish compliance was made, this 
time successfully. However, the compliant attitude of the patient was only directed at the medicating process in accordance with the hospital rules. We may speculate that the motivation behind it was the doctor's decision to continue her treatment in the hospital despite her previous devious behavior, but there is no conclusive data on the matter. A.A's condition gradually improved and about 5 weeks later she was discharged from the hospital and her relatives finally took her home.

\subsection{Commentary}

This case is one of the majorities one can find, because nowadays, the focus is shifted toward medication use and not what happens to patients after they leave the hospital with a prescription. A lot more could be uncovered if the usual framework was reshaped. For example, the role of the husband's habits and behavior was never placed into the social context of family status. Was current family environment a part of the reasons for the relapse or was it a different personal psychological experience unknown to the family members? Were the lower education, the language, and cultural gap, all of them creating communication difficulties between patient and doctor, a valid excuse to use the safe, well-known evidence-based approach? And to what degree would the outcome have been different, had there a crosscultural connection between the doctor and the patient been established?

These questions may remain unanswered in the context of the current case, but their explanation may change the general framework we confide ourselves in as doctors. It is difficult to establish a productive collaboration with a patient whose behavior is influenced not only by their current condition but also by lack of education, language barrier, and different cultural views.

In the contemporary materialistic world, Roma people often face discriminative attitude, since their lifestyle differs significantly. Subsequently, it is a common phenomenon for mental health issues as a contributing factor to such attitude to be underrated and reported at a delayed stage, which has a negative impact on the treatment outcome [1]. Inside this frame of reference, we cannot be certain whether the perception of the impaired mental state of this young woman as a stigma did not influence the time of diagnosis and the course of treatment.

The status of Roma people within the society is not only a reflection of their cultural traditions but also a possible explanation for their different behavior of illness. A study by Vorvolakos et al. showed higher prevalence of some psychiatric disorders in Roma women, compared to their majority counterparts, further supplemented by the discrepancy in the structural/categorical distribution of psychiatric disorders between these groups, namely, the low frequency of more severe diagnoses such as psychosis or bipolar disorder among Roma women [2]. The same study presented a significant contrast in the socioeconomic circumstances of Roma women, which are characterized with higher social pressure in terms of familial issues such as child raising and lack of cultural independence.

Considering these findings in the context of sources of distress sheds new light on the observed behavior of illness of both Roma patients in general and our 
particular case. However, since the family dynamics was neither investigated, nor targeted in the therapeutic approach, the specific cultural and transcultural interactions were not clarified. Thus, we can only speculate about the role of the family customs and traditions as a source of distress for A.A. and as a predicament for sustaining the patient's compliance.

These are only a few of the numerous more examples why the patient's cultural beliefs and personality traits are at the core of a successful treatment. Unlike all other specialties, psychiatric disorders are hardly ever cured purely by means of psychopharmacology. And even though great progress has been made in understanding the mechanisms through which psychopharmacological medications act in the processes and causes underlining psychiatric conditions, what defines the success of treatment is still a matter of providing care for the person behind the disease in the context of their culture and traditions.

\subsection{The Values Arising in this Story}

If we review this case within the framework of a conventional evidence-based medical approach, we may conclude that it was handled correctly-a thorough medical and family history was obtained, followed by examination and various tests, a therapeutic approach was specified and subsequently altered, according to the course of the disorder, and a significant improvement of the patient's condition was observed as the outcome. If, however, we step outside this conventional medical frame and change the perspective, we may see an entirely different image, one less colorful and at best half-finished.

When one reads this story between the lines, they will see many questions left unanswered. For example, is it possible for A.A. to perceive her illness as an escape from her addicted husband? If so, is it her choice to stay with him and what are the fundamental values behind such a decision? What triggered the excessive alcohol consumption of her husband-was it her condition or something else? And whatever the reason is, have his values deteriorated beyond repair or can he still be a source of support, not fear, for his wife? The answers to these could have provided yet another tool to understand and treat A.A's illness, but they were not obtained.

When it comes to the treatment plan, even more questions arise, because it remained strictly evidence-based, purely pharmacological and value-free. Do we attribute this decision to the doctor's values or to the framework that medicine understood as not more than a science has created? Perhaps it is a combination of both, since following the guidelines is safe and well grounded; however, the choice whether to stay within these boundaries belonged to the doctor alone. Yet, the choice to let A.A. stay and continue the treatment even after she was subject to disciplinary discharge is extremely value-laden, because it was not made due to following a set of rules, but for the single purpose to help.

Another "side effect" of the evidence-based treatment of a woman with such a value-laden cultural inheritance is that it can never establish the necessary trust and respect between the doctor and the patient, and hence, it couldn't be and remained unsuccessful in that her compliance continued only within the four walls of the 
hospital with her treatment remaining purely pharmacological and dependent on the constant supervision of medical personnel. It is not medications that the Romani believe in, but their rituals for purification. Therefore, compliance with the treatment may have been ensured, if it was supplemented with a method directed to changing those particular psychological barriers, set by the patient's beliefs (for example, hypnosis or a religious ritual for purification). It is worth adding that the psychiatric department where A.A. was hospitalized has previous experience in applying methods such as hypnosis, but it was not included in her documented treatment plan.

Some of the reasons for her failed treatment are worth emphasizing. This was essentially because A.A.'s symptoms were not perceived as culturally influenced. Thus, the therapeutic approach undertaken couldn't be and remained unsuccessful because it was directed only toward those symptoms and their probable biological underpinnings, and not to their cultural cause. Consequently, The medical specialists deprived themselves of a valuable accessory therapeutic mechanism by neglecting the significance of investigating the family dynamics within their Roma cultural context and how including the family members in the diagnostic and treatment processes might have influenced the course of the illness. If in this case, the relatives perceived the doctor's efforts to observe and value their traditions as part of the therapy, while at the same time, they were well acquainted with the signs and symptoms of the disorder and how it was likely to progress, they might have supported the patient's treatment not only by ensuring that she was regularly medicated but also by taking part in the arrangement of any culturally appropriate rituals that would have satisfied the patient's psychological attitude and understanding. When the family members realize the association between conflicts, emotions, and symptoms (the importance of specific cultural, regional, ethnic, etc., language expressions such as "nerves, nerve attack, crisis"), they may at least try to change their attitude to the patient and be more accepting, considerate, tolerant, forgiving, less critical, and demanding.

The reactively chosen medical approach may be understood as an expression of the "value blindness" concept of values-based practice. The doctor listens to the patient, trying to find a combination of symptoms, defining a particular diagnosis, but at the same time fails to recognize the values driving these symptoms. This "values blindness" may reflect an existing educational gap and/or a language barrier.

It is very important to recognize the necessity of maintaining a balance between science and values not only in psychiatry but also in medical practice as a whole, because the cause, development, and treatment of every medical condition are deeply influenced by cultural inheritance and personal values.

\subsection{The Influences of Culture on this Story}

We know that somatization and conversion are frequently resultant superficial manifestations of suppressed/repressed emotional conflicts, specifically related in most cases to interpersonal (family, relatives, and friends) conflicts and tension, especially when suffering individuals are suggestible and alexithymic or when these are 
culturally accepted idioms of distress. For the Romani, the origins of illness are "pollution", lack of good fortune, etc. It is their firm belief that the larger a person is, the healthier, luckier, and happier that person will be [3]. In this context, the onset of the patient's disorder is related to the loss of weight that in her culture, it is perceived as loss of good fortune. Most of her symptoms were most likely related to this loss and were a presentation of a culturally specific distress.

If we look at the history of the Romani people, we might find ourselves astonished at how much of their current differences can be rooted in their life in the past. This is a nomadic population who was often persecuted and discriminated against (discrimination is still a relevant hindrance for their integration to western culture nations), and for that reason, their most sacred relationships are those within the so-called extended family. This can also relate to their lower educational status. It was essential for their survival in the past to stay united and supportive of each other during their long journeys from place to place, mastering abilities that could provide them with the daily bread, which can hardly fit in the contemporary view of proper education. This has also led to the immense diversity of the professed religions of the Romani people, which, however, does not undermine the influence these religious and familial identities have on their well-being and their perception of such [4]. Also, religious beliefs and values are known to have a major effect on mental health and behavior in every individual [5] .

The Roma traditions and beliefs have a deep influence over their behavior that in turn they are trying to change because nowadays they lead a settled life. It may be impossible for an outsider to fully comprehend the complexity of the meaning of their traditions because these incorporate both the endowment of their ancestors and the endeavor to preserve their sacred and rich history within the new world they are trying to fit in.

\subsection{Conclusions}

The main purpose of medicine and psychiatry is to not only treat the symptoms of a disease but also to improve the patient's quality of life, which necessitates the application of an integrative approach combining state-of-the-art scientific findings with the ancient cultural inheritance of every human being. In order to accomplish a paradigm shift toward personalized and values-based medical approaches, we need to realize the complex cross-cultural interactions in the doctor-patient relationship. Moreover, we should try to gain a unique perspective on every single patient as opposed to the current tendency to reduce all individuals to the common denominator of their disease and expand our range of knowledge beyond conventional therapeutic guidelines.

Acknowledgements The story of A.A. was kindly submitted by Valentina Milkovska, M.D.State Psychiatric Hospital, Pazardzhik, Bulgaria. 


\subsection{Guide to Further Sources}

For a more extensive account of the elements of values-based practice and the crosscultural characteristics of the Romani people, see Ref. 2.

\section{References}

1. Lee EJ, Keyes K, Bitfoi A, Mihova Z, Pez O, Yoon E, Masfety VK. Mental health disparities between Roma and non-Roma children in Romania and Bulgaria. BMC Psychiatry. 2014;14:297.

2. Vorvolakos T, Samakouri M, Tripsianis G, Tsatalmpasidou E, Arvaniti A, Terzoudi A, Livaditis M. Sociodemographic and clinical characteristics of Roma and non-Roma psychiatric outpatients in Greece. Ethn Health. 2012;17(1-2):161-9.

3. Vivian C, Dundes L. The crossroads of culture and health among the Roma (Gypsies). J Nurs Scholarsh. 2004;36(1):86-91.

4. Dimitrova R, Chasiotis A, Bender M, van de Vijver FJ. Collective identity and well-being of Bulgarian Roma adolescents and their mothers. J Youth Adolesc. 2014;43(3):375-86.

5. Bhugra D. Commentary: Religion, religious attitudes and suicide. Int $\mathrm{J}$ Epidemiol. 2010;39(6):1496-8.

Open Access This chapter is licensed under the terms of the Creative Commons Attribution 4.0 International License (http://creativecommons.org/licenses/by/4.0/), which permits use, sharing, adaptation, distribution and reproduction in any medium or format, as long as you give appropriate credit to the original author(s) and the source, provide a link to the Creative Commons license and indicate if changes were made.

The images or other third party material in this chapter are included in the chapter's Creative Commons license, unless indicated otherwise in a credit line to the material. If material is not included in the chapter's Creative Commons license and your intended use is not permitted by statutory regulation or exceeds the permitted use, you will need to obtain permission directly from the copyright holder.

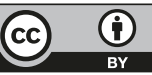

